\title{
Açai Juice as Contrast Agent in MRCP Exams: Qualitative and Quantitative Image Evaluation
}

\section{Kátia Elisa Prus Pinho ${ }^{1^{*}}$}

https://orcid.org/0000-0002-3542-9283

\section{Antonio Carlos Pinho 2}

https://orcid.org/0000-0002-4014-8072

Julio Cesar Pisani ${ }^{3}$

https://orcid.org/0000-0001-5165-0300

\section{Carlos Alberto Goedert 4}

https://orcid.org/0000-0002-5255-4844

\section{Andreia Magri Gusso 4}

https://orcid.org/0000-0003-0716-1002

\section{Pedro Miguel Gewehr ${ }^{1^{\star \star}}$}

https://orcid.org/0000-0002-9694-7906

${ }^{1}$ Graduate Program in Electrical and Computer Engineering (CPGEI) and ${ }^{* *}$ Department of Electronics, Federal University of Technology-Paraná (UTFPR), Brazil;

${ }^{2}$ Department of Electrical Engineering, UTFPR, Brazil;

${ }^{3}$ Professor of Gastroenterology, Paraná Federal University (UFPR), Brazil;

${ }^{4}$ Cetac: Diagnostic Clinic, Brazil.

Received: 2016.08.25; Accepted: 2019.02.21.

*Correspondence: katiaprus@utfpr.edu.br; Tel.: +55-41-33104680 (K. E. P. P.)

\section{HIGHLIGHTS}

- Açai juice was employed as a contrast agent in MRCP exams.

- A protocol was established for the contrasts administration.

- Images of MRCP were compared qualitatively and quantitatively (Image J). 
Abstract: This works aims to assess images obtained with administration of açai juice as compared to a manufactured standard iron oxide-based contrast employed as negative oral agents in Magnetic Resonance Cholangiopancreatography (MRCP), employing qualitative and quantitative evaluation. The research was developed with 64 patients submitted to MRCP exams (on 2 days) in a clinic of Curitiba city (Brazil). First (day 1), a manufactured iron oxide-based contrast (A) was offered and later (day 2), açai juice (contrast B) was given to patients. Radiologists (R1 and R2) evaluated the images, classifying them by a score (1-4). In order to have a quantitative assessment, Image $\mathrm{J}$ free software was employed generating plots of gray levels against distance of a chosen area of the bile duct interest region. Evaluating images for contrast $A, R 1$ furnished an average score of 3.52 and R2 of 3.27. For contrast B, R1 provided 3.44 and R2 3.38. Both evaluators considered image quality with contrast $A$ adequate for 62 patients. $R 1$ considered adequate for 62 and R2 for 60 patients when using açai juice. By taking same images for all patients with Image $\mathrm{J}$, a quantitative analysis was obtained, resulting correlation coefficient of 0.986 between average curves of contrasts A and B. Thus, açai juice is an adequate alternative as contrast agent in MRCP exams. Image $\mathrm{J}$ was employed as a new method for quantitative investigation of image quality, presenting good agreement with medical opinion.

Keywords: Açai contrast agent; iron oxide contrast; magnetic resonance cholangiopancreatography; image quality; image $\mathrm{J}$.

\section{INTRODUCTION}

Magnetic Resonance Cholangiopancreatography (MRCP) exams make use of a negative contrast to identify and visualize organs such as pancreas and gallbladder. In the images, contrast agents can avoid the overlapping of these organs [1]. The procedure is useful for detection of pancreatitis, cholelithiasis, biliary sludge, pseudo pancreatic cysts, tumors, and others. Images acquired by magnetic resonance with natural contrasts are very similar to those acquired with manufactured ones, according to previous work done by other authors [2,3].

For a juice to be effective as contrast agent in MRCP exams, some features are required [4], besides in addition, reducing the signal intensity from the stomach and duodenum and it must improve the bile duct and gallbladder views. In general, the contrast agent should: contain ferromagnetic substances such as iron (Fe) [5] and, change the recovery and decline times (T1 and $\mathrm{T} 2$, respectively) $[4,6]$. Several natural contrasts have been used in MRCP exams including orange, lemon, pineapple, blueberry and, açai juices $[3,4,7,8]$. One of the most promising natural agents is açai since good results were obtained in tests (in vitro) on magnetic resonance imaging for various T1 and T2 weighting sequences [9]. Among the advantages of a natural agent, it does not cause adverse reactions and is more palatable [8] than manufactured agents. In clinical terms, to be successful in MRCP exams, a juice must reduce signal strength between stomach and duodenum, being able to produce a medical report with diagnostic quality.

Although there are papers about the use of juices as MRCP contrast agents [3,4], it was not found publications about standardization procedure and administration protocol of the juices [4,5]. Image evaluation is achieved from several ways in clinics and hospitals by means of medical analysis or using some computational tool $[10,11,12]$. These can help to identify lesions, tumors or alteration of the organ or tissue being examined. Image evaluation can present inherent limitation due to the technique employed or analysis made by different personnel and equipment.

Since there is a great amount of subjectivity on the assessment of the acquired images by medical teams, depending on the previous experience of each evaluator, there is an opportunity to explore the use of Image $\mathrm{J}$ free software $[13,14]$ in order to produce a new quantitative analysis of the images and to compare them to medical evaluation. Thus, the main objective of this study was to assess quality of MRCP images, obtained with the administration of the promising açai juice $[8,15]$ and a manufactured iron oxide-based 
contrast employed as negative oral contrast agents, by means of medical evaluation and with the use of Image J software [14].

\section{MATERIAL AND METHODS}

\section{Contrast agents}

In this work 2 types of contrasts were employed: a natural one (açai juice) and a manufactured iron oxide-based contrast. Considering the use of natural contrasts on previous works $[4,6,9]$, a natural juice prepared from açai (Euterpe oleracea) pulp was employed. The juice was produced with the fruit pulp $(100 \mathrm{~g})$ and mixed with pure water (about $100 \mathrm{~mL}$ ) by using a blender, without any sugar added. The juice (natural contrast agent) contained approximately $36 \mathrm{~g}$ of carbohydrates per $100 \mathrm{~g}$ of pulp, corresponding to the amount of natural sugar in the fruit [15].

The juice was prepared (room temperature) on the same day of the exam, and it remained in the refrigerator about 4 hours before administration.

The manufactured iron oxide (175mg of Fe per liter of water) based contrast [5] was used as specified for MRCP exams and acquired from Guebert Ltda. Each acquired flask contained $300 \mathrm{~mL}$ of the contrast ready to be used (iron oxide diluted in water).

\section{MRCP exam protocol}

As patients would need a medical report, the exam was started (first day) after the ingestion of the manufactured iron oxide-based contrast (referred as A) with the sequence of full abdomen, followed by MRCP. On the second day, 24 horas after the administration of contrast $A$, there was the ingestion of açai juice (referred as contrast $B$ ), and the MRCP sequence was also performed. Since the ingestion of the contrasts was oral, the elimination is fast [16]. Thus, there was no interference of contrast $A$ with $B$, since the organs of interest for the exams excrete most of the substances within one hour after ingestion [16].

For patient preparation, it was determined 3 hours of absolute fast [17]. The determination of the fasting time was established by means of the physicians participating of the research. On the first day, when the patient arrived at the clinic, he/she was directed to the preparation room, the fasting time was confirmed and the manufactured contrast $(A)$ was fractionated in 2 doses of $100 \mathrm{~mL}$, one dose was given after the patient responded the anamnesis, and another was given 10 minutes later. Just after the second dose, the MRCP exam was started. On the second day, the procedure was similar, only changing the contrast, i. e., administration of açai juice (B) in 2 doses of $100 \mathrm{~mL}$ was given as above.

The anamnesis contained anthropometric data, history of allergy, use of medicines, pre-existing diseases (as diabetes and hepatitis), surgeries, etc. In case of a diabetic patient, glucose measurement in blood was performed before and after ingestion of contrasts.

Doctors gave support to the patients in the days of exams, but the type of contrast administered each day was kept secret. Therefore, the study was double-blind and prospective $[4,7,18]$.

The images were acquired with a 1.5T MRI system [8] from General Electric Company (GE), model HDXT with 12 channels, GE Healthcare Advantage workstation running Centricity DICOM (Digital Imaging and Communications in Medicine) Viewer version 3.0 software, and were saved in the filing system and communicating images (PACS-Picture Archiving and Communication System) [19], in a clinic of Curitiba city (Brazil). The usual MRCP acquisition protocol was used: localizer (LOC) in 3 orthogonal planes (PL) following single-shot (SS), fast spin echo (FSE) in apnea (LOC 3 PL SSFSE Apnea); radial colangio $[9,20]$ and axial lava $\mathrm{T} 1$ without fat $[21]$ for the two days of exams.

\section{Patient selection}

The selection of patients was made at the Clinics Hospital of Parana Federal University (UFPR), which receives patients from the metropolitan region of Curitiba city (via Health 
Units). The clinical research was developed during a period of 6 months, in the outpatient clinics of non-alcoholic esteatopathy, alcoholic liver disease and fat and biliary routes diseases. The selected patients needed to perform the MRCP exams and they were volunteers for the research.

The study was approved by the Ethics Committee of Federal University of Technology-Paraná (UTFPR) by number 02.520.512.0.00005547 and a free and informed consent form (ICF) was released. Patients who agreed to participate received the ICF and appropriate guidance on the MRI examination [8].

Patients who participated met the criteria: age between 18 and 80 years for both genders, need to perform MRCP exams and present pancreaticobiliary disease and/or alteration in liver ducts $[16,17]$ being in treatment or follow-up in the referred hospital and, ingest the contrast agents orally and accomplish fasting of 3 hours in the 2 days of the exams. Also, patients should lie down, not have claustrophobia nor hearing difficulties.

Exclusion criteria included pregnancy or suspected pregnancy as well as patients who could not ingest juice and/or contrast agent or had some difficulty as: not being able to perform fasting, making use of devices such as pacemakers, stents, aneurysm clips, intraocular foreign bodies, cochlear implants, prosthetic/ pins in any region of the body which could cause artifacts [22].

\section{Medical images analysis}

For the analysis of images [8] there was the collaboration of 2 radiologists identified as $\mathrm{R} 1$ and R2, both with large experience in MRCP image diagnosis.

To analyze the effects of contrasts, scores 1-4 were used, having as reference the action of the contrast in places where it must make effect (stomach and duodenum). Score 1 means that there is a hyper intensity of the stomach and duodenum signals, thus it is not possible to evaluate these structures. Score 2: evaluation takes place partly when it is possible to visualize the structures. Score 3: the hyper intensity of the signal does not hinder the analysis of structures. Score 4: there is no signal hyper intensity between the stomach and duodenum, which makes clearer the MRCP exam $[8,9]$.

The evaluation of the image quality was performed by doctors filling a patient individual form which contained: image quality classification for contrasts $A$ and $B$ as excellent, good, fair and poor; if the acquired image for contrasts $A$ and $B$ could replace each other; and if the contrasts were suitable and efficient for issuing a medical report [23].

Also, a new quantitative evaluation method based on Image $\mathrm{J}$ free software, 16-bits version, was employed [14] and it can furnish the intensity of pixels against distance of a chosen area of an image. By taking a section of interest from the image, one can limit the anatomical region of the structure to be analyzed. After, the selected area (with $x$ and $y$ values) is transferred to an Excel® file and a plot is produced with gray levels (pixel intensities) against distance (width of the bile duct region for this study) in $\mathrm{mm}$ [24].

\section{Statistical analysis}

Data analysis of patients was performed with the items in the anamnesis and medical evaluations, in order to assist in verifying the behavior of the variables [25]. Subsequently, it was evaluated the concordance of responses of doctors through Kappa [26] statistics, aimed at testing the degree of concordance (reliability and precision), and to assess the values obtained. If $>0.75$, it indicates an excellent result. Values $<0.40$ are considered low or if they become between 0.40 and 0.75 are of median concordance [26].

\section{RESULTS}

\section{MRCP Protocol}

Altogether 86 patients were invited to the study and 64 (31 females, 33 males) participated in the exams. The implemented MRCP protocol allowed $1536(64 \times 2 \times 12)$ acquisitions of sequences of radial colangio for all patients in the two days of exams. 
The principal features for the patients [8] are shown in Table 1. Ages were in the range $\geq 18$ and $\leq 78$ years for females and, $\geq 27$ and $\leq 70$ for males. The average age was 54.6 years, with women presenting higher age (56.4 years) than men (52.9 years).

Table 1. Main features of participants of the MRCP protocol.

\begin{tabular}{ccc}
\hline & Age (years) & Average Age (years) \\
Female & $\geq 18 \leq 78$ & 56.4 \\
Male & $\geq 27 \leq 70$ & 52.9 \\
& & \\
Female & Frequency & Percentage \\
Male & 31 & 48.4 \\
\hline
\end{tabular}

\section{Medical Evaluation}

For the assessment, each evaluator chose between the sequences of images, two of them, one for each type of contrast. The images had to show the region of the biliary tract with quality and reduce the signal of the stomach and duodenum allowing a medical report. Table 2 shows the frequency of medical scores attributed by evaluators R1 and R2. For score 1, no image was chosen. For score 2, on the 1st day R1 evaluated 1 patient and R2 4, on the 2 nd day 4 were evaluated by R1 and 8 by R2. For score 3, on the 1 st day 29 were for $\mathrm{R} 1$ and 39 for R2, and on the 2nd day 28 were for R1 and 24 for R2. Finally, for value 4, on the 1st day they were 34 (R1) and 21 (R2), and on the 2 nd day evaluations were 32 for each doctor.

Analysis was performed to evaluate the correlation between assessments on the 2 days of tests [8]. For the 1st day, the coefficient was 0.521, indicating a moderate agreement between evaluators. The $95 \%$ confidence interval for the coefficient ranged from 0.363 to 0.679 . On the 2 nd day the coefficient was 0.674 , also indicating a moderate concordance for two doctors. The $95 \%$ confidence interval ranged from 0.521 to 0.828 .

There was little difference among doctors on evaluating images: R1 maintained the average score of 1 st day 3.52 and, $R 23.27$. On the 2 nd day, $R 1$ was 3.44 , and $R 2$ was 3.38 . Thus, $\mathrm{R} 2$ seemed to be more rigorous than R1, assigning lower scores to images.

Table 2. Image Scores for Contrasts A and B as Attributed by Evaluators 1 and 2.

\begin{tabular}{ccccc}
\hline & \multicolumn{2}{c}{ Evaluator (R1) } & \multicolumn{3}{c}{ Evaluator (R2) } \\
\cline { 2 - 5 } Scores & \multicolumn{2}{c}{ Contrast } & Contrast \\
\cline { 2 - 5 } & A & B & 0 & B \\
\hline 1 & 0 & 0 & 4 & 0 \\
2 & 1 & 4 & 39 & 8 \\
3 & 29 & 28 & 21 & 24 \\
4 & 34 & 32 & 64 & 32 \\
Total & 64 & 64 & 64 \\
\hline
\end{tabular}

Considering the evaluation performed by doctors [8], there was a question related to image quality as to the suitability of the contrast (physical and chemical characteristics, including good digestive acceptance, not causing adverse reactions and not stimulating the intestinal peristalsis) [3] for an efficient report. Also, there was another question for efficiency: elimination of high intensity signals from the stomach and duodenum and, capacity to provide a complete view of the bile tree and pancreatic duct [14] for the contrasts. As for adequacy, evaluators agreed that for 62 patients (96.9\%), both contrasts were 
adequate and only for $2(3.1 \%)$ were not. This percentage of $3.1 \%$ may be linked to the technique of examination, no cooperation of the patient in image acquisition and diseases that hindered the visualization of anatomical areas of the MRCP exam. Considering efficiency, evaluator R1 kept the two contrasts for 62 patients (96.9\%) as effective and for 2 $(3.1 \%)$ not. For R2, the efficiency of contrast A occurred with 62 patients $(96.9 \%)$ and with 2 $(3.1 \%)$ did not. For contrast B, the efficiency occurred with 60 patients (93.8\%) and with 4 $(6.2 \%)$ did not.

\section{Image J Analysis}

In order to apply the Image $\mathrm{J}$ software, patients who presented the same score for the two evaluators were chosen (both days). Patients were identified by gender (F: female and, $\mathrm{M}$ : male) in addition to a sequential number [8]. Figure 1 ( $a$ and $b$ ) shows an example of high standard images as assessed by $\mathrm{R} 1$ and $\mathrm{R} 2$. Both images received score 4 from both doctors on 2 days. The images were acquired with administration of contrasts $A$ (manufactured) and B (açai juice) for the radial colangio sequence of patient F16 (which presented same score for both evaluators). It is seen, similarly, in both images that the contrasts canceled the stomach and duodenum signals, showing the completion of the bile duct. Image $\mathrm{J}$ software was employed with Figure 1 ( $a$ and $b$ ) to obtain an image quantitative analysis by taking a chosen section of the bile duct (lower third since it is of great interest for image quality) [27]. The arrows indicate the chosen areas of the duct lower third of about $60.58 \mathrm{~mm}$ (width) $\times 3.22 \mathrm{~mm}$ (height) as indicated by doctors for both contrasts to build Figure 1c. Figure 1c shows gray levels (pixels intensity) against distance where 4 anatomical regions are shown for both contrasts. Arrow 1 indicates the duodenum, 2 the bile duct, 3 the pancreatic tract and, 4 the pancreas head. 


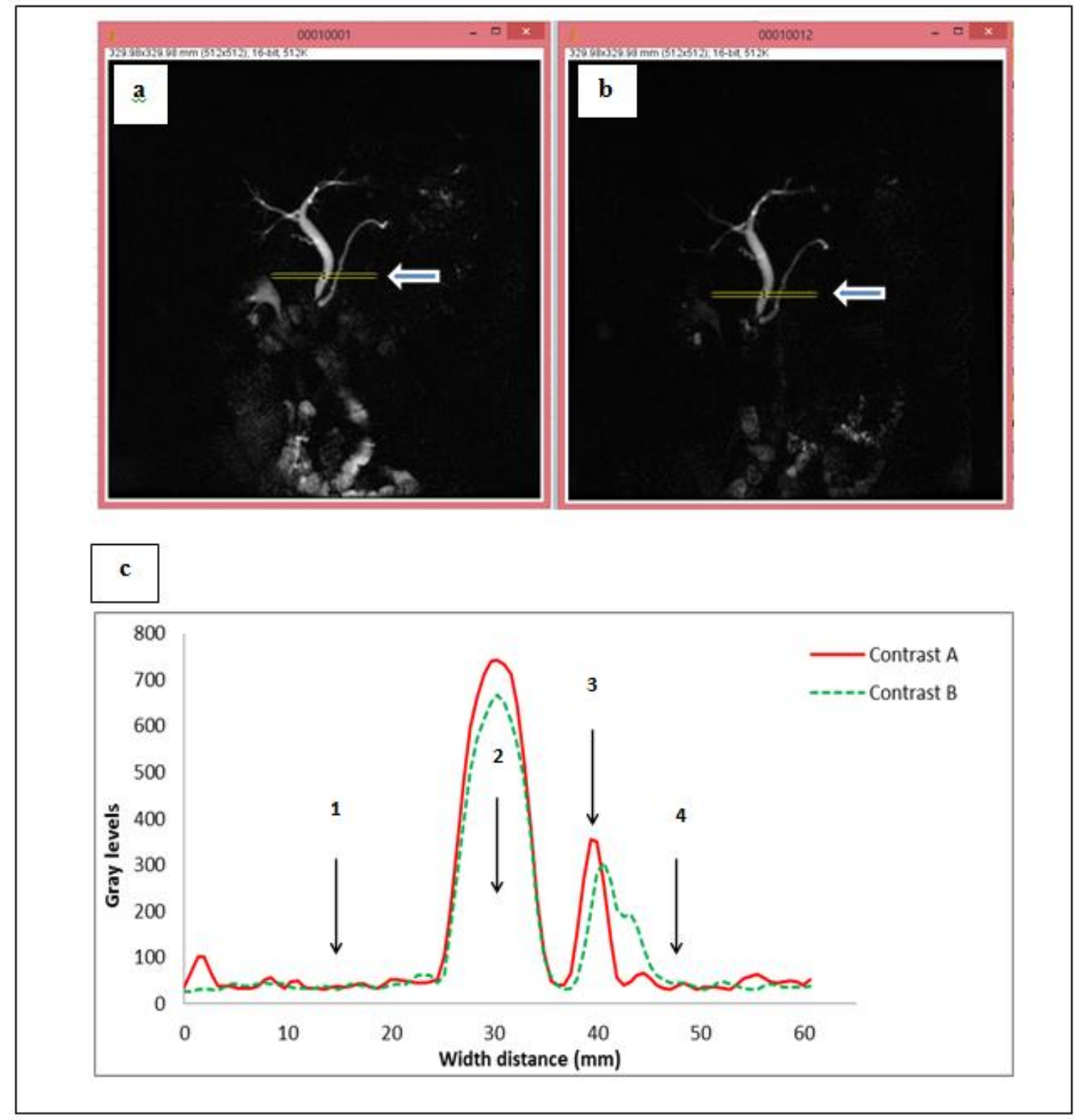

Figure 1. Images of the colangio radial sequence of patient F16 that received same score (4), where: (a) obtained with contrast A and; (b) contrast B; (c) Curves of gray levels against distance for both contrasts as obtained with Image J, with arrows indicating regions of (1) duodenum, (2) bile duct, (3) pancreatic tract and, (4) pancreas head.

Gray levels of contrast $A$ had values from 31.4 to 102 for region 1, 49.8 to 743 for region 2, 40.8 to 356.4 for region 3 and, 30.2 to 65.6 for region 4 . Considering contrast $B$, values obtained were from 26.2 to 62.4 (region 1), 44.6 to a 668.8 (region 2), 30.4 to 306.4 (region 3 ) and, 30.4 to 192.4 (region 4). For image quality, the duodenum region should be darker (hypo signal) and the bile duct should be brighter (hyper signal). As one can see, the intensity levels have a kind of asymmetric function behavior with the mean intensity levels for both contrasts of the left region (1) being lower (45.9) than the right region (3 e 4) with 77.4.

One can also estimate the section of the bile duct at the lower third of about $9 \mathrm{~mm}$, which is compatible with anatomical standards for adult males and females (usually there is little difference between genders).

Figure 2 shows average curves for scores 2, 3 and 4 obtained for female $(F)$ and male (M) patients with contrast A (as Table 2). Figure 2a was built for evaluator R1 and Figure 2b for R2. 
(a)

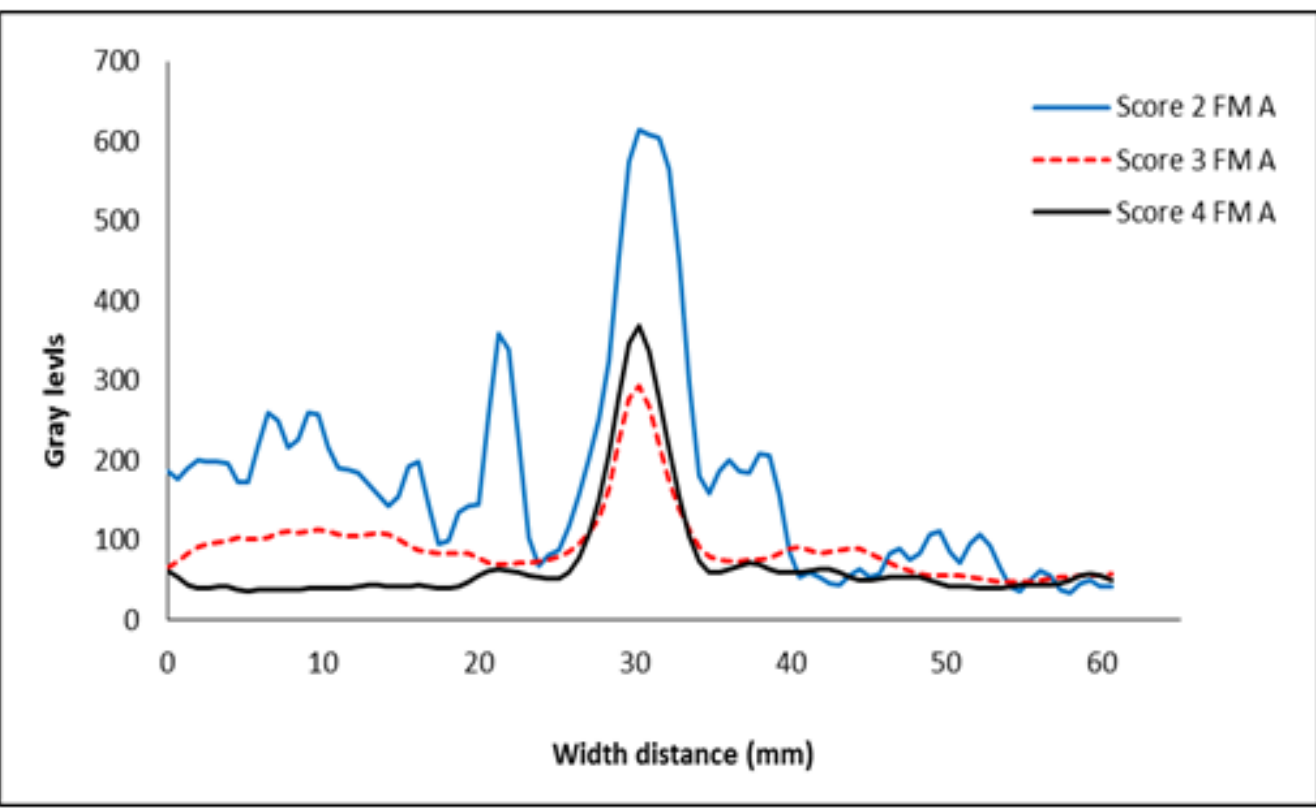

(b)

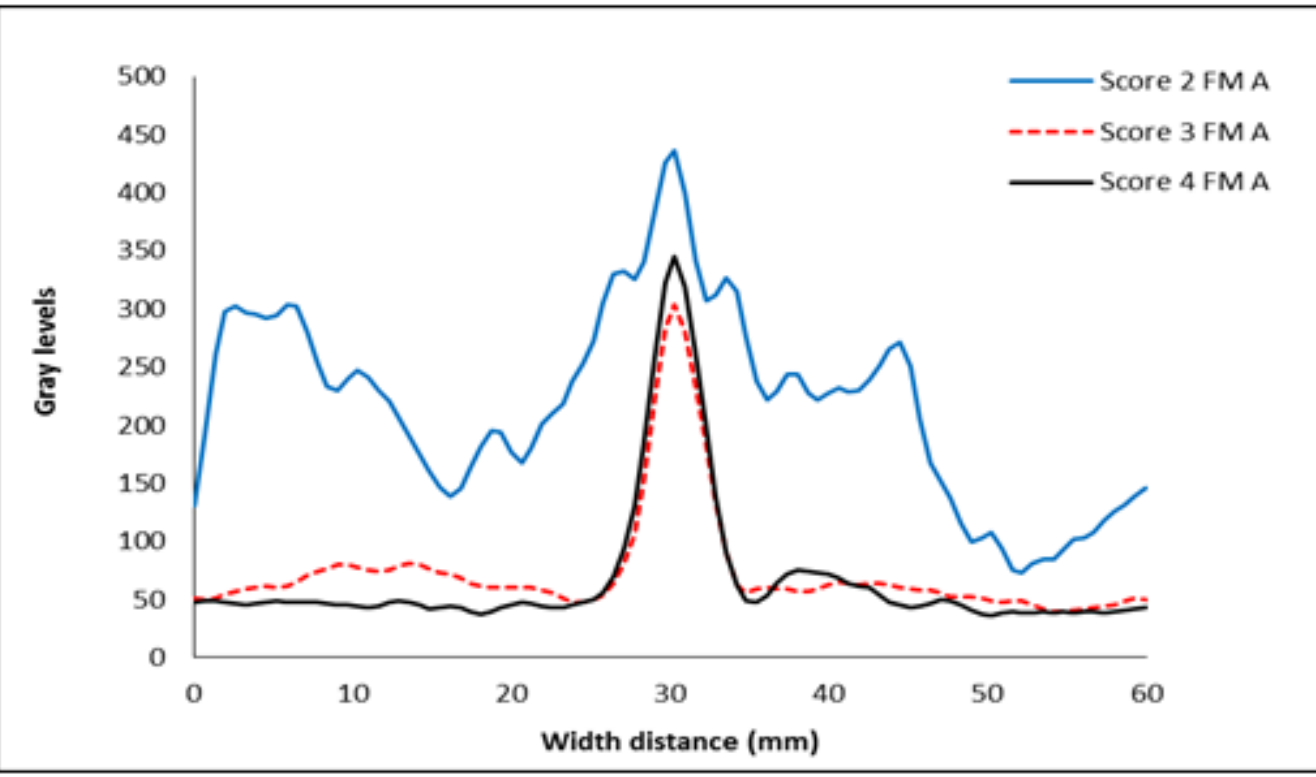

Figure 2. Mean curves for all patients (females and males) for each score with contrast A (as Table 2). a) Evaluator R1; b) Evaluator R2.

Comparing mean curves with scores 3 and 4, a correlation coefficient of 0.897 is obtained between them for R1 and 0.975 for R2, showing little quantitative difference between scores 3 and 4 for each evaluator, according to Image $\mathrm{J}$ software.

Figure 3 shows average curves for scores 2, 3 and 4 obtained for female $(F)$ and male (M) patients with contrast $\mathrm{B}$ (as Table 2). Figure $3 \mathrm{a}$ was built for evaluator R1 and Figure $3 \mathrm{~b}$ for R2. 
(a)

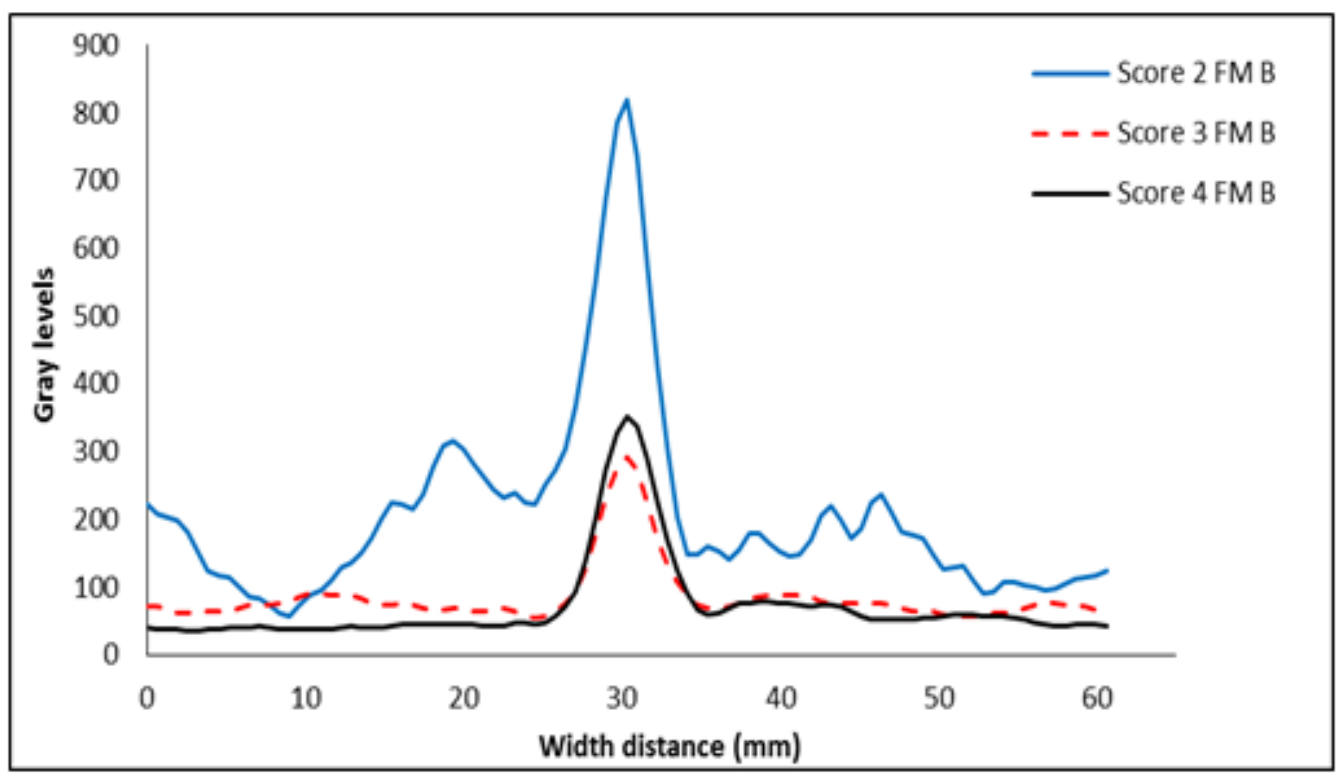

(b)

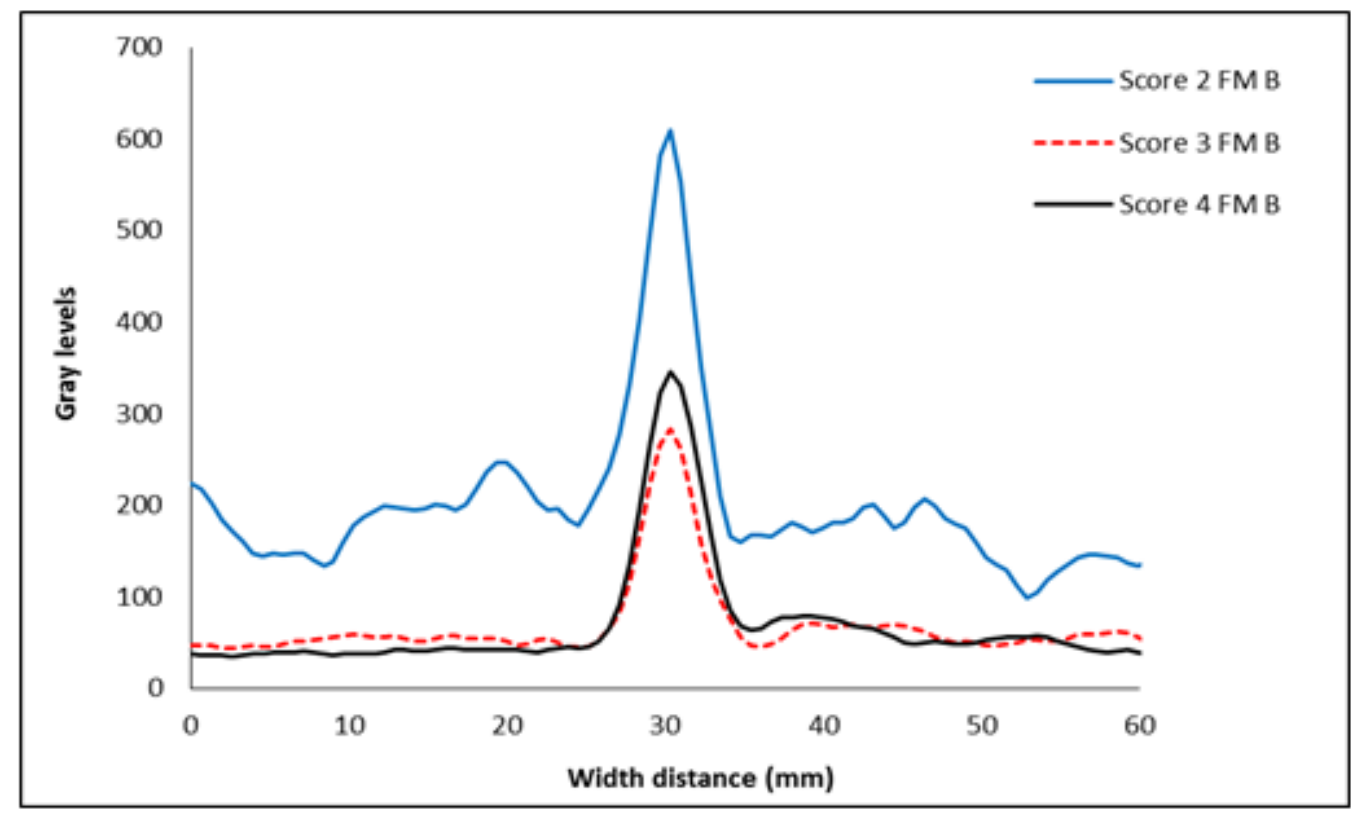

Figure 3. Mean curves for all patients (females and males) for each score with contrast B (as Table 2). a) Evaluator R1; b) Evaluator R2.

Comparing mean curves with scores 3 and 4 , a correlation coefficient of 0.970 is obtained between them for R1 and 0.983 for R2, showing very little quantitative difference between scores 3 and 4 for each evaluator, according to Image $\mathrm{J}$ software.

Table 3 presents mean intensities of each region as taken from curves for contrast $A$ (Fig. 2a-R1 and Fig. 2b-R2) and contrast B (Fig. 3a-R1 and 3b-R2) per score considering radiologists ( $\mathrm{R} 1$ and $\mathrm{R} 2$ ) for all patients. The regions were taken as shown on Figure 1c. Regions 3 and 4 were grouped together (Region 3-4). 
Table 3: Mean intensities of regions per score considering evaluators $R 1$ and $R 2$ for contrasts $A$ and $B$, as taken from Figures 2 and 3.

\section{Contrast A}

Scores

\begin{tabular}{ccccccccccccc} 
& \multicolumn{2}{c}{ Region 1 } & \multicolumn{2}{c}{ Region 2 } & \multicolumn{2}{c}{ Region 3-4 } & \multicolumn{2}{c}{ Region 1 } & \multicolumn{2}{c}{ Region 2 } & \multicolumn{2}{c}{ Region 3-4 } \\
& $\mathbf{R 1}$ & $\mathbf{R 2}$ & $\mathbf{R 1}$ & $\mathbf{R 2}$ & $\mathbf{R 1}$ & $\mathbf{R 2}$ & $\mathbf{R 1}$ & $\mathbf{R 2}$ & $\mathbf{R 1}$ & $\mathbf{R 2}$ & $\mathbf{R} 1$ & $\mathbf{R 2}$ \\
$\mathbf{2}$ & 189.1 & 229.3 & 353.2 & 331.9 & 86.9 & 162 & 177.8 & 187.3 & 438.5 & 344.7 & 148.2 & 160.1 \\
$\mathbf{3}$ & 92.6 & 64.8 & 159.1 & 147.8 & 66.6 & 53 & 71.5 & 51.5 & 153.6 & 142.5 & 71.8 & 58 \\
$\mathbf{4}$ & 45.4 & 47.5 & 179.8 & 189.8 & 53 & 51.5 & 40.6 & 39.9 & 178.8 & 175 & 58.9 & 56.7 \\
\hline
\end{tabular}

The chosen regions are in accordance to Figure 1c. Region 1 ( 0 up to $24.49 \mathrm{~mm}$, duodenum); Region 2 (25.13 up to $34.8 \mathrm{~mm}$, bile duct); Region 3-4 (35.45 up to $60.58 \mathrm{~mm}$, pancreatic tract and pancreas head).

All images acquired can be used with Image $\mathrm{J}$, including the cases with associated morbidity that presented ascite and/or alteration of abdominal wall. Figure 4 shows a case (M37) where the image shows artifacts (arrows 1) on both acquisitions as obtained with contrasts A (Figure 4a) and B (Figure 4b).

Arrows 2 (Fig. 4a and Fig. 4b) indicate the regions where Image $\mathrm{J}$ was employed to generate the curves in Figure 4c. The regions appear to be dark in Figure 4 ( $a$ and b) but when using Image $J$ the curves obtained (Figure 4c) show some relatively high level of gray for all sectors including regions 1 and 4 with levels above 100. These anatomic structures were partially identified by both doctors. The images received score 3 with R1 (contrasts $A$ and $\mathrm{B}$ ) and score 2 with $\mathrm{R} 2$ (both contrasts). Using Image $\mathrm{J}$, one can see that the high level of gray is due ascite and not caused by contrast in the duodenum. Medical reports related moderate ascite, some chronic liver diseases, portal hypertension and suspected hepatic cancer which could support the artifacts found and the spreading of liquids in the cavity close to the selected areas for Image J.

\section{DISCUSSION}

In our study there was a firm determination of a protocol that would meet the requirements and the clinical condition of the patient. As for contrasts ingested both manufactured and açai juice, the 64 patients were able to ingest the established amount of $200 \mathrm{~mL}$. Thus, the MRCP exam protocol was suitable mainly due to regularity of fasting and correct ingestion of contrasts.

Quality of medical images for some authors $[7,9,28]$ showed a significant difference in healthy female volunteers who received 2 different types of substances, one administered with empty and another with full stomach. In other studies [3,9] medical evaluations were performed with and without contrast to MRCP, with the amount ranged from 100 to $350 \mathrm{~mL}$ and $2 \mathrm{~mL} / \mathrm{kg}$, and administering different contrasts (pineapple juice with $\mathrm{Gd}$, lemon or orange juice, manganese chloride tetra hydrate, ferric ammonium citrate [29]. In our study, medical evaluations were made for 2 contrasts for all patients, maintaining administration of same amount of $200 \mathrm{~mL}$ of contrast a day. Also, the analysis of images was always with contrasts, i.e., the patient had 2 tests on different days, respecting a 10 min interval between doses. The standardization of the contrast volume provides security to the teams involved as well as time reduction without loss of quality and better finding of anatomical areas. 


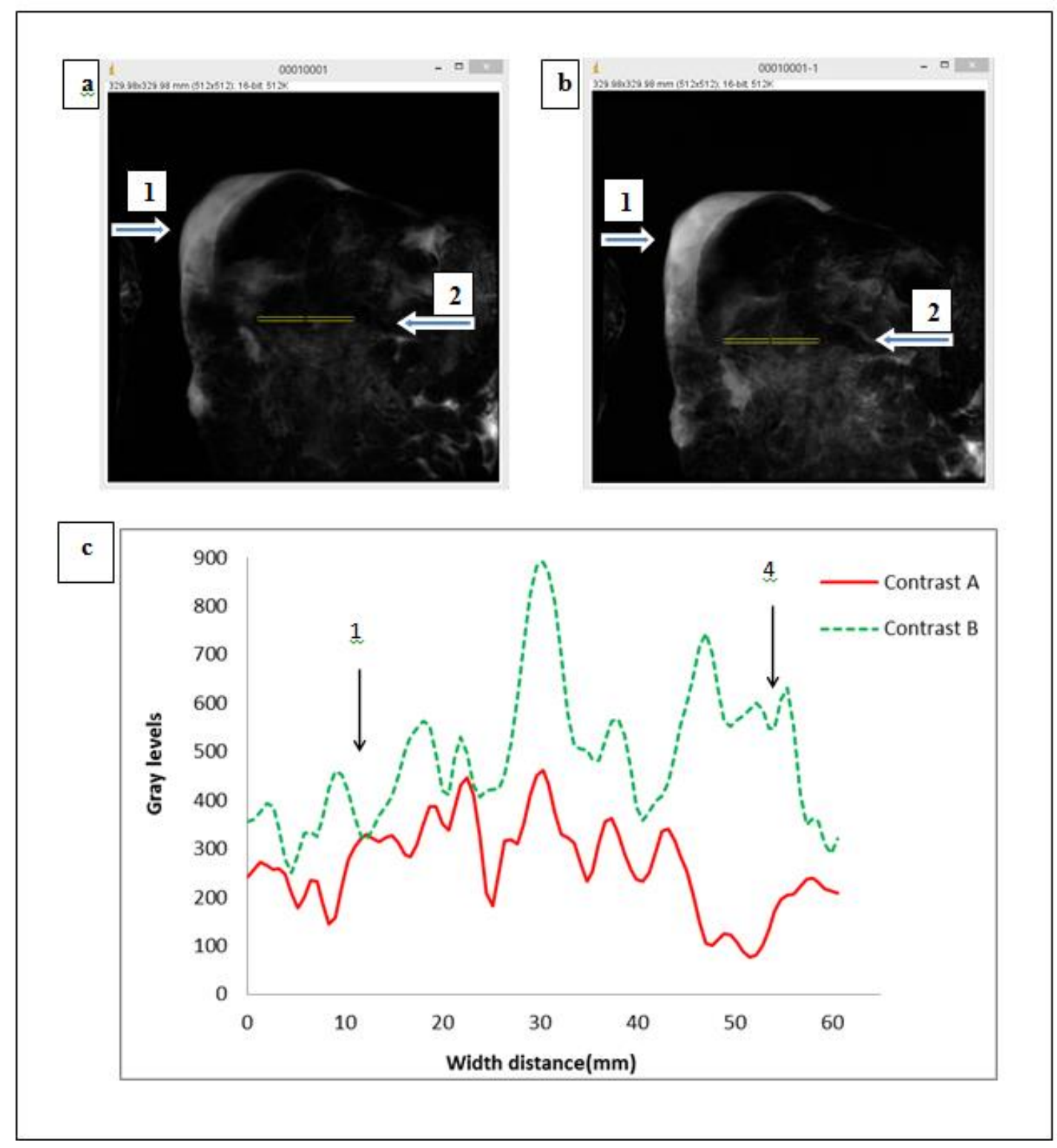

Figure 4. MRCP Images for patient M37. a) Contrast $A$, arrow 1 shows artifact regions and arrow 2 the region selected for Image J; b) Contrast B; (c) Curves of gray levels against distance for both contrasts as obtained with Image J. Arrows 1 and 4 show increased gray level.

Contrasts were kept blind in our study as in other works [4, 7] and, also a question was included on adequacy and efficiency of natural and manufactured contrasts to improve the medical report. It was observed that for evaluator R1 the 2 contrasts showed the same results for efficiency (96.9\%) and $\mathrm{R} 2$ was more rigorous, evaluating contrast B $93.8 \%$ efficient [8].

According to Bates et al. [1], the MRCP exam needs a clear view of the disease by means of the image. The use of a negative contrast is essential and has allowed in our study the identification or confirmation of existing diseases such as (frequency and type): 11 hepatitis, 15 liver cirrhosis, 6 liver steatosis, 2 liver tumors, 12 altered thyroid function, 3 pancreatitis, 25 diabetes and other as: kidney, ascite, human immunodeficiency virus (HIV), esophagitis, etc. In the process of investigation and diagnostic confirmation, there were 7 patients. As diseases may be common to the same patient (i.e., diabetes and hepatitis) someone could have one or more associated diseases.

Image $\mathrm{J}$ software was employed previously to analyze MR brain images of Alzheimer disease people and healthy volunteers $[30,31]$ and to compare the findings with clinical 
exams. Here, a new application was found for Image $\mathrm{J}$ and then employed to compare MRCP images obtained with 2 contrasts and evaluated by medical staff by taking gray levels in the regions of interest (Figure 1c).

Figures 2 and 3 have shown characteristic patterns of gray levels for determined regions of each curve. Thus, regions 1 presented lower intensities for increasing scores. The same is valid for regions 3-4. This is expected since these regions must be darker for better image quality. Region 2 presented higher intensities for higher scores, which is also expected in order to obtain more visible and brighter bile ducts. The very high values obtained for score 2 are exceptions and can be explained by the few images of patients evaluated as score 2.

Finally, the 8 images of patients that received score 2 with contrast B from evaluator R2 were difficult to visualize bile ducts, being considered low quality images. In practice patients presented several pathologies with liquids in the abdominal cavity or other problems close to the duodenum, etc. Thus, Figure 4 is another example that confirms image medical assessment with the quantitative analysis provided by Image $\mathrm{J}$.

\section{CONCLUSION}

As described, by using a proposed and tested protocol, açai juice showed a similar action of a manufactured contrast. The effectiveness of natural contrast was equal or greater than $93.8 \%$, and for the manufactured one was equal to $96.9 \%$, since both darkened the signal from stomach and duodenum and provided appropriate conditions for a MRCP quality medical report [8]. Açai juice and its manufactured counterpart were employed with a reduced fasting time $(3 \mathrm{~h})$, being compatible for exams at different times and at any time of the day, and in several age groups (infants, children, adults and elderly). More, patients with difficulty to make a long fast can benefit from the natural agent, since it does not cause collateral effects and/or discomfort. Thus, one can consider açai juice as an efficient alternative to manufactured contrasts, including its use for patients with hepatic and/or gastric alterations.

Contrasts $A$ and $B$ quantitatively presented similar values of gray levels in the regions of interest and high correlation coefficients for all scores provided by evaluators R1 and R2 (including the examples presented (patients F16 and M37)). The curves of gray level intensities against width of the bile duct and region presented characteristic patterns for each region of the images, being possible to link these patterns to image quality. The quantitative results obtained almost completely agree with the opinion of evaluators $\mathrm{R} 1$ and $\mathrm{R} 2$ and at the same time open an opportunity to further studies to establish a protocol using an automatic and precise new analysis tool for medical image reports.

Funding: This research was partially funded by Fundação Araucária from Paraná State (Brazil), project 355/2012.

Acknowledgments: Statistical Laboratory Applied (LEA) of UFPR; Clinics Hospital of UFPR and, Diagnostic Clinic of Curitiba city where the work was carried out; UTFPR students, Max Vinicius de Carvalho Souza and Nhynéya Kharoline da Silva Rocha, for their help to produce the juices as well as assisting the patients.

Conflicts of Interest: The authors declare no conflict of interest.

\section{REFERENCES}

1. Bates, D.D.B.; Lebedis, C.A.; Soto, J.Á.; Gupta, A. Use of magnetic resonance in pancreatobiliary emergencies. Magn Reson Imaging Clin N Am 2016, 24, 433-448.

2. Morita, S.; Ueno, E.; Masukawa, A.; Suzuki, K.; Fujimura, M.; Hirabayashl, N.; Kojima, S.; Kitajima, K.; Kaji, Y. Prospective comparative study of negative oral contrast agents for magnetic resonance cholangiopancreatography. Jpn J Radio/ 2010, 28 (2), 117-122.

3. Chu, Z.Q.; Ji, Q.; Zhang, J.L. Orally administered lemon/orange juice improved MRCP imaging of pancreatic ducts. Abdom Imaging 2010, 35(3), 367-371. 
4. Fraga, T.C.; Araujo, D.B.; Sanchez, T.A.; Elias, J. Jr.; Carneiro, A. A.O.; Oliveira, R.B.; Rosa, M. B. Euterpe olerácea (açai) as an alternative oral contrast agent in mri of the gastrointestinal system: preliminar results. Magn Reson Imaging 2004, 22, 389-393.

5. Lumirem oral suspension. Available online: http://www.shijiebiaopin.net/upload/product/201481321502822.pdf (accessed on 10 March 2019).

6. Westbrook, C.; Roth, C.K.; Talbot, J. MRI in practice, 4th ed.; Wiley-Blackwell: Oxford, England, 2011.

7. Bittman, M.E.; Callahan, M.J. The effective use of acai juice, blueberry juice and pineapple juice as negative contrast agents for magnetic resonance cholangiopancreatography in children. Pediatr Radiol 2014, 44, 883-887.

8. Pinho, K. E. P.; Pinho, A.C.; Gewehr, P. M.; Gusso, A. M. Image Quality in Magnetic Resonance cholangiopancreatography exams: study between açai juice and a manufactured contrast agent. Proceedings of the Brazilian Conference on Biomedical Engineering (CBEB), Búzios, Brazil, 21-25 October 2018; SBEB: Búzios, Brazil, 2018.

9. Riordan, R.D.; Khonsari, M.; Jeffries, J.; Maskell, G.F.; Cook, P.G. Pineapple juice as a negative oral contrast agent in magnetic resonance cholangiopancreatography: a preliminary evaluation. Br J Radiol 2004, 77, 991-999.

10. Babos, M.; Schwarcz, A.; Randhawa, M.S.; Marton, B.; Kardos, L.; Palkó, A. In vitro evaluation of alternative oral contrast agents for MRI of the gastrointestinal tract. Eur Radiol 2008, 65, 133-139.

11. Duarte, J.A.; Furtado, A.P.A.; Marroni, C.A. Use of pineapple juice with gadopentetate dimeglumine as a negative oral contrast for magnetic resonance cholangiopancreatography: a multicentric study. Abdom Imaging 2012, 37, 447-456.

12. Oliveira, R.S.M.F.; Oliveira, F.A.M.; Pinheiro, H.S. Programming Image J software for automatic selection of immunohistochemistry marked areas stained by dab chromogen and counter-stained by hematoxylin. Rev Int Est Exp 2010, 2(3), 76-80.

13. Kestelman, F.P.; Souza, G.A.; Thuler, L.C.; Martins, G.; Freitas, V.A.R.; Canella, E.O. Breast Imaging Reporting and Data System - BI-RADS ${ }^{\circledR}$ : positive predictive value of categories 3,4 and 5. Radiol Bras 2007, 40(3), 73-77.

14. Image J. Available online: https://imagej.nih.gov/ij/ (accessed on 12 December 2015).

15. Açai mais fruta. Available online:.http://www.maisfruta.com.br/pt/produtos/19/acai-fino (accessed on 10 May 2015).

16. Guyton, A.C.; Hall, J.E. Guyton and Hall Textbook of Medical and Physiology, Saunders Elsevier: Philadelphia, USA, 2011.

17. Kato, J.; Kawamura, Y.; Watanabe, T.; Okada, S.; Cho, K.; Ishihara, M. Examination of intra-gastrointestinal tract signal elimination in MRCP: combined use of T1- shortening positive contrast agent and single-shot fast inversion recovery. J Magn Reson Imaging 2001,13,738-743.

18. Agresti, A. Categorical data analysis, John Wiley \& Sons: Hoboken, USA, 2014.

19. Marques, P.M.A.; Caritá, E.C.; Benedicto, A.A.; Sanches, P.R. RIS/PACS integration at "Hospital das Clínicas de Ribeirão Preto": a web based solution. Radiol Brasil 2005, 38(1), $37-43$. 
20. Fukukura, Y.; Takumi, K.; Higashi, M.; Shinchi, H.; Kamimura, K.; Yoneyama, K.; Tateyama, A. Contrast-enhanced CT and diffusion- weighted MR imaging: Performance as a prognostic factor in patients with pancreatic ductal adenocarcinoma. Eur Radiol 2014, (83), 612-619.

21. Pinheiro, Y.L.S.; Costa, R.Z.V.; Pinho, K.E.P.; Ferreira, R.R.; Schuindt, S.M. Effects of iodinated contrast agent, xylocaine and gadolinium concentration on the signal emitted in magnetic resonance arthrography: a samples study. Radiol Bras 2015, 48(2), 69-73.

22. Westbrook, C. Handbook of MRI Technique, 3th ed.; John Wiley \& Sons: Oxford, England, 2014.

23. Mazzola, A.A.; Herdade, S.B.; Koch, H.A.; Carvalho, A.C.P. Acceptance testing protocol for magnetic resonance imaging systems. Radiol Bras 2005, 38(3), 195-204.

24. Tang, X.N.; Berman, A.E.; Swanson, R.A.; Yenari, M.A. Digitally quantifying cerebral hemorrhage using Photoshop $\AA$ and Image J. J Neurosci Methods 2010, 190, 240-243.

25. Montgomery, D.C.; Runger, G.C. Applied Statistics and Probability for Engineers, 5th ed.; John Wiley \& Sons: Hoboken, USA, 2014.

26. Sim, J.; Wright, C.C. The Kappa statistic in reliability studies: use, interpretation, and sample size requirements. Phys Ther 2005, 85(3), 257-268.

27. MRI. Image processing and analysis with ImageJ and MRI cell image analyzer. Available online: http://www.mri.cnrs.fr/datas/fichiers/articles/60/183.pdf (accessed on 11 July 2016).

28. Sanchez, T.A.; Elias, J.Jr.; Colnago, L.A.; Troncon, L.E.A.; Oliveira, R.B.; Baffa O.; Rosa, M. B. Clinical feasibility of açai (Euterpe oleracea) pulp as an oral contrast agent for magnetic resonance cholangiopancreatography. J Comput Assist Tomogr 2009, 23 (5), 666-670.

29. Espinosa, M.G.; Sosa, M.; Rodríguez, L.M.L.; Córdova, T.; Alvarado, J.B.; Rodríguez, M.A.; Aguilera, J. A. R.; Ortíz, J. J.; Barrios, F. A. Blackberry (Rubus spp.): a pH-dependent oral contrast medium for gastrointestinal tract images by magnetic resonance imaging. Magn Reson Imaging 2006, 24, 195-200.

30. Passoni, S.; Borges, F.S.; Pires, L.F.; Saab, S.C.; Cooper, M. Software Image J to study soil pore distribution. Ciênc Agrotec Lavras 2014, 38 (2), 122-128.

31. Jaba, L.S.; Shanthi, V.; Singh, D.J. Estimation of Hippocampus volume from MRI using Image J for Alzheimer's diagnosis. A J Med Biol Sci 2011, 1(1), 15-20. 